Federico Mainardi

Carlo Lisotto

Claudia Palestini

Paola Sarchielli

Ferdinando Maggioni

Giorgio Zanchin

\section{Headache attributed to airplane travel (“airplane headache"): first Italian case}

Received: 16 January 2007

Accepted in revised form: 18 April 2007

Published online: 11 June 2007

F. Mainardi

Headache Centre, Neurological Unit,

SS Giovanni e Paolo Hospital,

Castello 6777, I-30122 Venice, Italy

Tel.: +39-041-5294417

Fax: +39-041-5294555

e-mail: federico.mainardi@ulss12.ve.it

C. Lisotto

Headache Unit,

San Vito al Tagliamento Hospital, Italy

C. Palestini

Department of Medicine,

Adria Hospital, Adria, Italy

P. Sarchielli

Headache Centre,

Department of Neurosciences,

University of Perugia, Perugia, Italy

F. Maggioni • G. Zanchin

Headache Centre,

Department of Neurosciences,

Padua University, Padua, Italy
Abstract A new form of headache, whose attacks seem to be stereotyped, has been recently reported; because of the peculiarity of its onset, strictly related to airplane travel, the name of "Airplane headache" was proposed. A total of 7 cases have been published. Here we present the first Italian one. Furthermore the revision of the clinical characteristics of each patient leads us to propose provisional diagnostic criteria.
Keywords Airplane headache • Barotrauma • Diagnostic criteria • Headache attributed to airplane travel

\section{Introduction}

The term "airplane headache" first appeared in 2004, indicating the case of a 28 year-old man suffering from severe headache associated only with airplane travel; in particular, he complained the onset of the pain during taking-off and landing of the aircraft [1]. Further six new similar cases were published in a recent paper: the pain was severe, usually uni- 
lateral in the fronto-temporal/orbital region, without relevant accompanying signs or symptoms [2]. We recently observed a young man who complained of a severe orbital pain strictly related with air travel.

Although the cases described, including the present one, are only eigth, the clinical features of the attacks are very similar; for this reason, we felt that a preliminary proposal of diagnostic criteria could be put forward.

\section{Case report}

A 23 year-old male complained of a very severe pulsating pain in the right retro-orbital and frontal region associated with ipsilateral tearing. The duration was $10-15$ minutes. $\mathrm{He}$ suffered from these attacks exclusively during air travel, 60-90 minutes after taking-off. He had travelled six times in the previous year and each time he presented an attack. He did not take any medication because of the short duration of the episodes. Previously, he had travelled by air for years without any problem. Medical history was not significant; in particular, nothing was reported that could possibly be related to rhinosinusitis. General and neurological exams were normal. Neuroradiological investigations (cerebral MRI and AngioMRI) did not show any abnormalities.

\section{Discussion}

The first case suffering from this syndrome was published in 2004: a 28 year-old man who complained in his early 20s the sudden onset of a severe pain in the frontal region and between the eyes, without any accompanying signs or symptoms; he had travelled previously by airplane without any problems. The pain, described as sharp jabbings, began within 10 minutes from taking-off, lasted about 20 minutes, and reappeared with the same features during landing. $\mathrm{He}$ referred similar episodes each time he flied in the following years [1].

A series of patients reporting a very close clinical syndrome has been recently published [2].

The main features of the cases already described, along with those of our present case, are reported in Table 1. This has been partially modified from Berlingen and Müngen [2], with the addition of age of onset, correlation with each fly, pain quality and intensity, presence of other primary headaches.

The common features of the headache associated with airplane travel are: 1 . Gender: all the patients were male; 2 . Unilaterality: seven (out of 8) patients complained a strictly unilateral pain; 3. Site of pain: the pain was consistently reported in the peri-orbital region, without secondary irradiation but in one case; 4 . Duration: each attack was short lasting, not exceeding 20 minutes, and resolved spontaneously; 5. Accompanying signs or symptoms: only one case reported the concomitant presence of stuffy feeling on the face area and omolateral nasal congestion; our patient had ipsilateral tearing; none complained of nausea, vomiting, phonophobia, photophobia or osmophobia, nor of other vegetative symptoms; 6. Age of onset and of observation: the peak of onset was 31.7 years (range 20-40), while the mean age of observation was 34.3 years (range 23-42); 7. Pain onset: the pain began more often during landing ( 5 cases out of 8 ), than during taking-off ( 2 cases, in our case 60-90 minutes after taking-off); in one case both during landing and taking off. Neurological examination and neuroimaging resulted normal in all the cases; 8 . Other primary headache: only two cases reported a concomitant primary headache, migraine without aura and retinal migraine respectively, which began years before the onset of the headache associated with airplane travel.

The aetiology and physiopathology of this form of headache are unclear: Berlingen and Müngen [2] in a detailed discussion suggest that a change of the environmental pressure, which may rapidly occur during taking off and landing, could lead to a barotrauma by different mechanisms; this could cause mechanic changes in the ethmoid cells, stimulation of the anterior ethmoid artery nociceptors and subsequent activation of the trigeminovascular system. The hypothesis proposed is suggestive and could explain the rapid onset of the pain, and the close temporal relation to a rapid change of the cabin pressure, which could occur during taking off and landing. However, our patient complained of pain regularly 60-90 minutes after taking-off, thus without any relationship to possible sudden changes in cabin pressure. Therefore, barotrauma might not completely explain the origin of this kind of headache, at least in our case.

For the same reason, the hypothesis of an influence of the meteorological conditions, supported by the observations that attacks recur frequently when landing on seaside airports [1], does not hold in our case.

Another point of interest to consider is the consistency of the attack at each flight. This was observed only in our case and in that of Atkinson and Lee [1]; it was not specified in two cases of Berlingen and Müngen, whereas the remaining four patients did not refer headache every time they travelled by air. This fact would not support the hypothesis of the presence of a subclinical condition affecting the ethmoid sinuses, as a predisposing factor to the triggering of the headache.

Apart from inferences on the possible causative factors and mechanisms of this peculiar clinical manifestation, the limited data present in the literature do not consent to achieve a conclusive nosography of this headache. However, considering the fairly stereotyped features of the attacks, it seems 


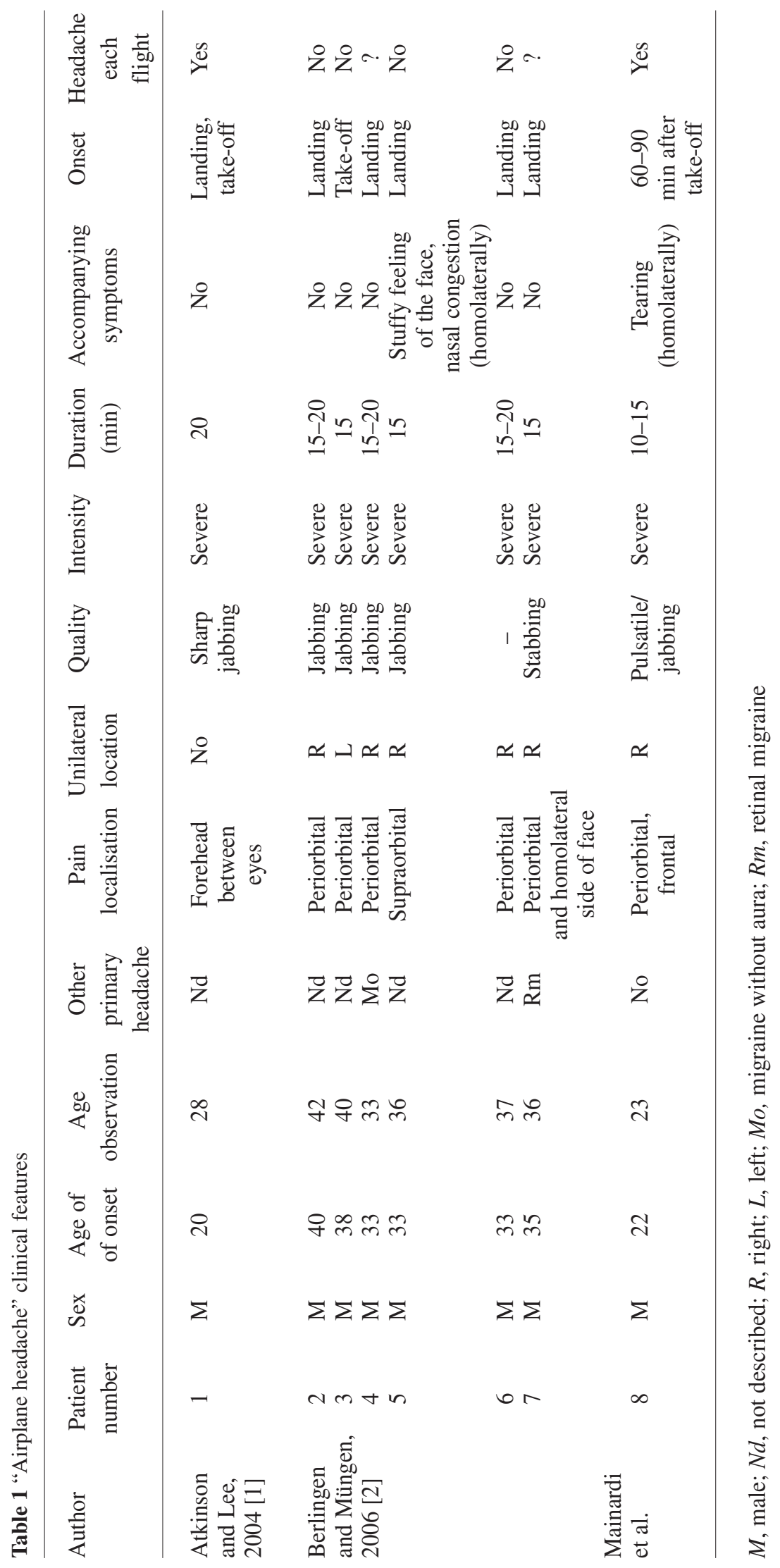


useful to suggest a proposal of provisional diagnostic criteria for this form of headache (Table 2). Furthermore, we suggest that airplane headache could be at the moment listed in the appendix of the International Classification of Headache Disorders [3], among other novel entities that have not been sufficiently validated by research studies.

Table 2 Headache attributed to airplane travel ("Airplane headache"): proposed provisional diagnostic criteria

A. At least 2 attacks, fulfilling criteria B-D

B. Pain during an airplane travel, lasting $<20$ minutes with at least two of the following:

1. Severe intensity

2. Jabbing or stabbing quality (pulsatility may occur)

3. Strict unilaterality

4. Periorbital location (forehead involvement may occur)

C. No accompanying symptoms (nasal congestion, stuffy feeling of face or tearing may occur omolaterally)

D. Not attributed to other pathologies

\section{References}

1. Atkinson V, Lee L (2004) An unusual case of an airplane headache.

Headache 44:438-439
2. Berlingen MS, Müngen B (2006) Headache associated with airplane travel: report of six cases. Cephalalgia 26:707-711
3. Headache Classification Subcommittee of the International Headache Society (2004) The International Classification of Headache Disorders. Cephalalgia 24[Suppl 1]:9-160 